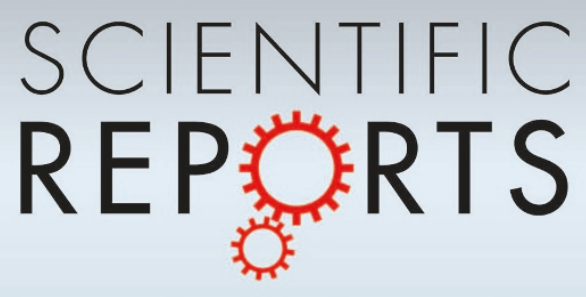

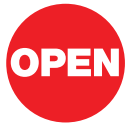

SUBJECT AREAS: MESENCHYMAL STEM

CELLS

BIOINSPIRED MATERIALS

BIOMEDICAL ENGINEERING

TRANSFECTION

Received

27 December 2012

Accepted

6 March 2013

Published

28 March 2013

Correspondence and requests for materials should be addressed to W.L.M. (wlmurphy@ wisc.edu)

\section{Inorganic coatings for optimized non-viral transfection of stem cells}

\author{
Siyoung Choi ${ }^{1}$, Xiaohua Yu ${ }^{2,3}$, Leenaporn Jongpaiboonkit ${ }^{4}$, Scott J. Hollister ${ }^{5,6,7}$ \& William L. Murphy ${ }^{1,2,3,8}$
}

\begin{abstract}
${ }^{1}$ Materials Science Program, University of Wisconsin-Madison, Madison, WI, USA, ${ }^{2}$ Department of Biomedical Engineering, University of Wisconsin-Madison, Madison, WI, USA, ${ }^{3}$ Department of Orthopedics and Rehabilitation, University of Wisconsin-Madison, Madison, WI, USA, ${ }^{4}$ Tissue Regeneration Systems, Inc., Ann Arbor, MI, USA, ${ }^{5}$ Department of Biomedical Engineering, University of Michigan, Ann Arbor, MI, USA, ${ }^{\circ}$ Department of Surgery, University of Michigan, Ann Arbor, MI, USA, ${ }^{7}$ Department of Mechanical Engineering, University of Michigan, Ann Arbor, MI, USA, ${ }^{8}$ AO Foundation Collaborative Research Center, Davos 87270, Switzerland.
\end{abstract}

"Biomimetic" approaches for heterogeneous growth of inorganic coatings have become particularly widespread in biomedical applications, where calcium phosphate $(\mathrm{CaP})$ mineral coatings are used to improve biomedical implants. Changes in coating properties can influence the effects of mineral coatings on adjacent cells, but to date it has not been practical to systematically vary inorganic coating properties to optimize specific cell behaviors. Here, we present an approach to grow CaP mineral coatings in an enhanced throughput format to identify unprecedented capabilities in non-viral gene delivery. Subtle changes in coating properties resulted in widely variable transfection, and optimized coatings led to greater than 10-fold increases in transgene expression by multiple target cell types when compared to standard techniques. The enhanced transfection observed here is substrate-mediated, and related to the characteristics of the local environment near the surface of dissolving mineral coatings. These findings may be particularly translatable to medical device applications.

norganic coatings have become important components in applications ranging from semiconductors to biomedical implants ${ }^{1,2}$. Over the past decade, a wide variety of studies have focused on using "biomimetic" approaches for heterogeneous growth of inorganic coatings ${ }^{3}$. The general biomimetic approach involves creating a surface with polar functional groups, incubating the surface in an aqueous solution loaded with inorganic mineral ions, and creating solution conditions that favor heterogeneous mineral nucleation and growth on the surface. This approach has become particularly widespread in biomedical applications, where calcium phosphate $(\mathrm{CaP})$ mineral coatings are used to enhance bone-implant healing ${ }^{4}$, to enhance mechanical strength of biomaterials $s^{5}$, and to control binding and release of drugs ${ }^{6}$ and genes ${ }^{7}$. Together, these previous studies emphasize the ability of biomimetic CaP coatings to improve biomedical implants. However, the properties of biomimetic $\mathrm{CaP}$ coatings are dependent on the solution characteristics during coating formation, and changes in the solution characteristics can result in significant changes in ionic composition, crystallinity, dissolution rate, porosity, and surface roughness of $\mathrm{CaP}$ mineral coatings ${ }^{8-13}$. Each of these parameters can influence the effects of mineral coatings on adjacent cells, but to date it has not been practical to systematically vary inorganic coating properties in the context of an individual study. Even though control of inorganic coating properties have been performed on the surface of cell culture plates this approach is not compatible with application of inorganic coatings on biomedical devices ${ }^{14,15}$. Here we present an approach to grow $\mathrm{CaP}$ mineral coatings in an enhanced throughput format, which we then use to identify unprecedented capabilities of $\mathrm{CaP}$ mineral coatings in non-viral gene delivery.

The factors that influence non-viral gene delivery are complex and interdependent. This complexity has led to a series of studies aimed at screening for organic polymers that can serve as gene delivery vectors. For example, combinatorial organic synthesis has been used to generate libraries of biodegradable polycations, allowing for significantly improved non-viral transfection ${ }^{16}$. We hypothesized that the same type of screening concept used to identify organic polycations could also be applied to inorganic materials. Specifically, we reasoned that inorganic coatings, formed via a biomimetic process, could be screened for their ability to enhance non-viral gene delivery. We specifically focused on CaP-based mineral coatings on bioresorbable polymers, which are FDA-approved and commonly used in biomedical applications. In addition, plasmid DNA (pDNA) is well-known to bind with high affinity to $\mathrm{CaP}$ minerals ${ }^{17}$ and calcium ions are a known co-factor in non-viral gene uptake ${ }^{18,19}$ and endosomal escape $^{20-22}$. Results demonstrate that it is possible to optimize CaP mineral coatings to achieve up to a 68 -fold 
increase in non-viral transfection of human mesenchymal stem cells when compared to standard techniques. This specific approach was then generalized to achieve enhanced transfection with multiple types of pDNA and multiple target cell types. The general concept may be used to screen for the effects of inorganic coating libraries on a variety of other phenomena.

\section{Results}

Experimental methods for optimizing transfection on mineral coatings. Recent studies have demonstrated that mineral surfaces can be useful platform not only supporting bone healing, but also enabling binding and release of biological molecules such as proteins, peptides and DNA. However, the broad ranges of parameters that can influence mineral properties make it difficult to optimize their effects on adjacent cells. To address this issue, we used an enhanced throughput approach to: efficiently form several mineral coatings; characterize mineral properties; and screen adjacent cell behavior (Fig. 1a). First we formed poly (lactide-co-glycolide) (PLG) films in 96-well plates, as PLG is among the most commonly used bioresorbable polymers in medical device design (Fig. 1a(1)). PLG was next hydrolyzed to expose carboxylate and alcohol groups on the surface, which promote heterogeneous mineral nucleation ${ }^{23}$ (Fig. 1a(2)). Mineral coatings were then synthesized on the hydrolyzed PLG films via incubation in a library of simulated body fluids (SBFs), which contained ionic constituents similar to blood plasma. Importantly, the well plate format allowed for efficient variation in
SBF contents, most notably the $\left[\mathrm{Ca}^{2+}\right],\left[\mathrm{PO}_{4}{ }^{3-}\right]$, and $\left[\mathrm{CO}_{3}{ }^{2-}\right]$ (Supplementary Table S1). Various modified SBF (mSBF) solutions were added into the wells (Fig. 1a(3)), and continuous mineral coatings were formed in all solutions after 6 days of incubation (Fig. 1b). The physical and chemical properties of mineral coatings were determined by Fourier-transformed infrared (FTIR), X-ray diffraction (XRD), and energy dispersive X-ray (EDS) analysis. To assess surface-mediated pDNA delivery from mineral surfaces, we then incorporated pDNA-Lipofectamine complexes within mineral coatings via a simple soaking process (Fig. 1a(4)). Cells were then seeded on the mineral coatings, and secreted luciferase in the cell culture medium was assayed to identify optimal mineral coating conditions and pDNA complex amounts for transfection (Fig 1a(5) and (6)). Note that throughout the manuscript, $\mathrm{mSBF}$ solutions are indexed as $\mathrm{A} \times$ or $\mathrm{A} \times-\mathrm{B}$, where $\mathrm{A}$ is the concentration of $\mathrm{Ca}^{2+}$ and $\mathrm{PO}_{4}{ }^{3-}$ in $\mathrm{mSBF}$ relative to those in blood plasma, and $\mathrm{B}$ is the $\left[\mathrm{CO}_{3}{ }^{2-}\right]$ in $\mathrm{mSBF}$. Color-coding throughout the figures indicates the $\left[\mathrm{CO}_{3}{ }^{2-}\right]$, with $4.2 \mathrm{mM}\left[\mathrm{CO}_{3}{ }^{2-}\right]$ (red), $25 \mathrm{mM}\left[\mathrm{CO}_{3}{ }^{2-}\right]$ (green), $50 \mathrm{mM}\left[\mathrm{CO}_{3}{ }^{2-}\right]$ (purple), or $100 \mathrm{mM}\left[\mathrm{CO}_{3}{ }^{2-}\right]$ (blue).

Modulating mineral coating properties. The coatings formed in $\mathrm{mSBF}$ solutions had intrinsically different mineral properties, including the extent of $\mathrm{CO}_{3}{ }^{2-}$ substitution, crystallinity, and composition (Fig. 2a, 2b, and 2c). FTIR analysis clearly indicates that the extent of $\mathrm{CO}_{3}{ }^{2-}$ incorporation into mineral structure was regulated by $\mathrm{mSBF}$ conditions. To evaluate the effect of $\mathrm{CO}_{3}{ }^{2-}$ incorporation

a

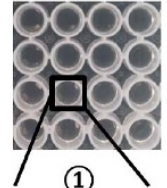

(2)

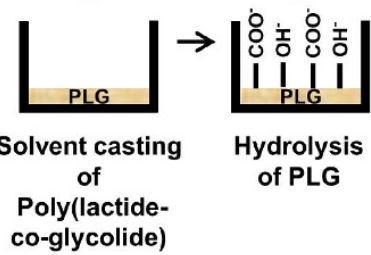

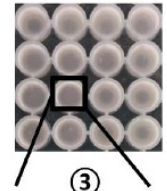

(3)
(4)

(5)
(6)

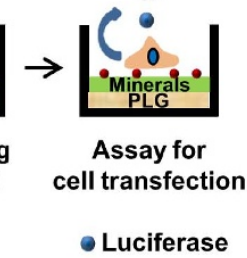

b

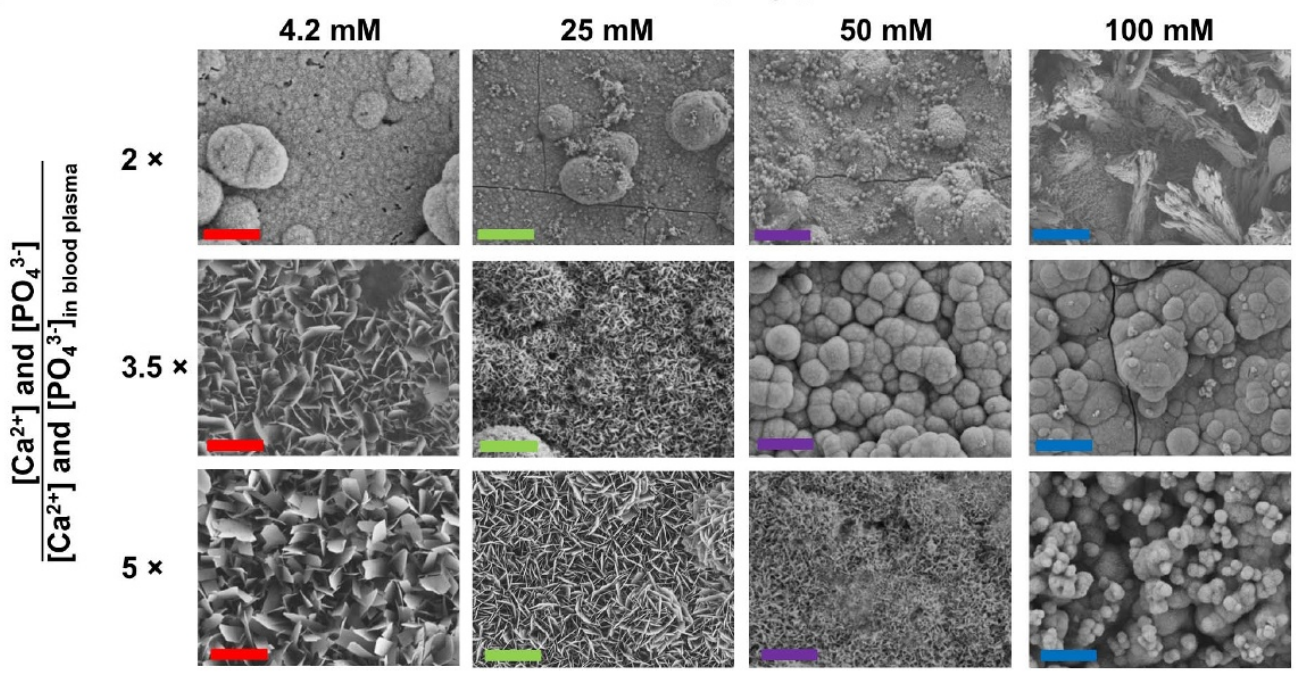

Figure $1 \mid$ Inorganic arrays for screening mineral-cell interactions. (a) Strategy for efficiently producing mineral coatings and screening mineral-cell interactions. Mineral coating is formed on hydrolyzed poly (lactide-co-glycolide) (PLG) using a library of modified simulated body fluids (mSBF) with various ions concentration. Then, stem cell transfection is measured by secreted luciferase after seeding on mineral coatings presenting pDNA complexes. (b) SEM images of mineral coatings after incubation in mSBF solutions containing various $\left[\mathrm{Ca}^{2+}\right],\left[\mathrm{PO}_{4}{ }^{3-}\right], \mathrm{and}^{2}\left[\mathrm{CO}_{3}{ }^{2-}\right](\mathrm{Scale} \mathrm{bars}, 10 \mu \mathrm{m})$. 
on mineral crystallinity, we used FTIR analysis to calculate the crystallinity index, which represents relative crystallinity between mineral coatings (Supplementary Fig. S1). The crystallinity index was modulated by changing the $\left[\mathrm{Ca}^{2+}\right],\left[\mathrm{PO}_{4}{ }^{3-}\right]$, and $\left[\mathrm{CO}_{3}{ }^{2-}\right]$ in the mSBF solutions (Fig. 2b), and the extent of amorphous phase in hydroxyapatite-like structure observed via XRD (Supplementary Fig. S2) corroborated the calculated differences in crystallinity index. In addition, $\mathrm{CO}_{3}{ }^{2-}$ incorporation into mineral structure increased calcium to phosphorus $(\mathrm{Ca} / \mathrm{P})$ ratio due to a substitution of $\mathrm{CO}_{3}{ }^{2-}$ for $\mathrm{OH}$ and $\mathrm{PO}_{4}{ }^{3-}$ (Fig. 2c). The effect of mineral property change on mineral stability was evaluated in cell culture media. Mineral dissolution kinetics were directly related to the mSBF solution conditions in which mineral coatings were produced. The degree of dissolution was proportional to $\left[\mathrm{CO}_{3}{ }^{2-}\right]$ in $2 \times \mathrm{mSBF}$ conditions, and this dependence was gradually diminished with the increase of $\left[\mathrm{Ca}^{2+}\right]$ and $\left[\mathrm{PO}_{4}{ }^{3-}\right]$ in $\mathrm{mSBF}$ solutions (Fig. 2d). These differences in mineral dissolution kinetics can be attributed to the extent of $\mathrm{CO}_{3}{ }^{2-}$ incorporation into the mineral structure during mineral formation (Fig. 2a), which has been previously shown to influence crystal size, crystallinity, and dissolution ${ }^{10,11}$. Increased $\mathrm{CO}_{3}{ }^{2-}$ incorporation led to spherulitic microstructure (Fig. $1 \mathrm{~b}(3.5 \times-50$ and $3.5 \times-100))$ as opposed to plate-like microstructure (Fig. $1 \mathrm{~b}(3.5 \times$ $-4.2,5 \times-4.2$, and $5 \times-25)$ ). The observed morphology changes result from inhibition of crystal growth by $\mathrm{CO}_{3}{ }^{2-}$ incorporation into the mineral structure. The changes in mineral crystallinity inversely correlated with the mineral dissolution rate in cell culture medium (Fig. 2e), as released $\mathrm{Ca}^{2+}$ was significantly increased when the crystallinity index was lower than 3 . These results are consistent with previous studies that correlate solution $\left[\mathrm{Ca}^{2+}\right],\left[\mathrm{PO}_{4}{ }^{3-}\right]$, and $\left[\mathrm{CO}_{3}{ }^{2-}\right]$ with resultant mineral coating structure, crystallinity, and dissolution kinetics ${ }^{8,10,11,24}$. Thus, mineral coatings formed on a common biomaterial (PLG) in an enhanced throughput format maintain properties that are consistent with previous studies performed in larger scale formats, and the well-plate format allows for efficient variation in inorganic material properties.

Optimized surface-mediated transfection by varying mineral coating properties. We hypothesized that systematic changes in mineral properties could provide a mechanism to optimize nonviral transfection. We reasoned that the region near the surface of dissolving mineral coatings would be characterized by high (and readily variable) local concentrations of pDNA complexes and calcium ions, which can serve as co-factors for non-viral gene delivery. As an initial step for surface-mediated transfection, pDNA complexes were immobilized on mineral coatings. Interestingly, there was no significant difference of binding efficiency between mineral coatings (Fig. 3a) even though mineral coatings have differences in several mineral properties like morphology (Fig. 1b) and pore size
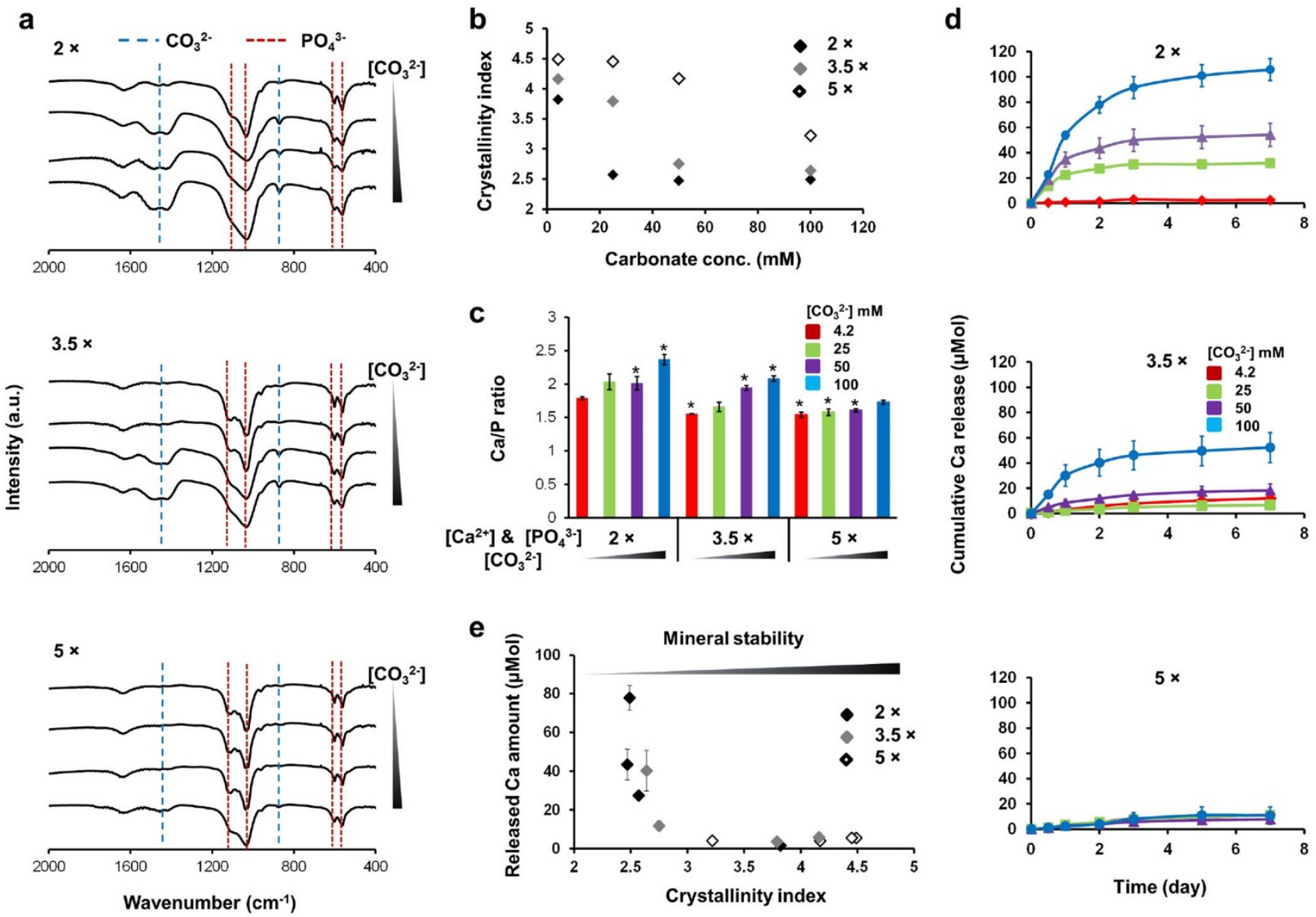

Figure 2 Systematic change of mineral coating crystallinity and its effect on mineral stability. (a) FTIR spectra of CaP mineral coatings. The peaks are associated with the $\mathrm{PO}_{4}{ }^{3-}(\ldots)$ and $\mathrm{CO}_{3}{ }^{2-}(--)$ groups. (b) The effect of mSBF solution characteristics on resulting mineral crystallinity index, which was calculated using FTIR analysis (Supplementary Fig. S1). (c) Ca/P ratio of mineral coatings. * indicates significant difference when compared with Ca/

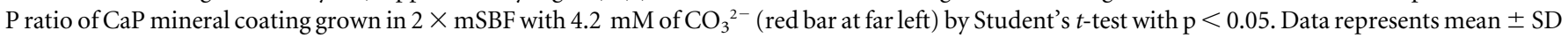
$(n=3)$. (d) Dissolution of mineral coatings formed in 12 different $\mathrm{mSBF}$ solutions. The change of $\mathrm{Ca}^{2+}$ amount in cell culture medium was measured at different time points. Data represents mean $\pm \mathrm{SD}(n=4)$. (e) The effect of relative crystallinity on mineral stability. The released Ca ${ }^{2+}$ amount after 2 days incubation in cell culture medium is plotted in terms of crystallinity index of mineral coatings. Data represents mean $\pm \operatorname{SD}(n=4)$. 
(Supplementary Fig. S3). This result may be due to the balance of mineral properties that influence pDNA complexes binding. Transfection of human mesenchymal stem cell (hMSC) on mineral coatings containing pDNA complexes indicates that hMSC transfection was dramatically influenced by the mineral coating stability (Fig. 3b), as measured by luciferase activity per cell viability (Supplementary Fig. S4). There was a direct dependence of luciferase expression on the $\left[\mathrm{CO}_{3}{ }^{2-}\right]$ in the $2 \times$ or $3.5 \times \mathrm{mSBF}$ solutions. These data suggest that transfection efficiency is dependent on mineral dissolution rate as the $\mathrm{CO}_{3}{ }^{2-}$ incorporation resulted in increased dissolution of coatings formed in $2 \times$ or $3.5 \times \mathrm{mSBF}$ solutions. In contrast, there was no significant dependence of luciferase expression on $\left[\mathrm{CO}_{3}{ }^{2-}\right]$ on mineral coatings formed in $5 \times \mathrm{mSBF}$, which did not change their dissolution rate with $\mathrm{CO}_{3}{ }^{2-}$ incorporation (Fig. 2d). Thus, transfection of hMSCs on pDNA complexes loaded mineral coatings
(Fig. 3b) correlated with the mineral dissolution rates (Fig. 2d), indicating that the level of luciferase activity can be controlled by varying mineral stability. Moreover, pDNA complex amount effect on transfection levels suggests a combinatorial approach for screening transfection on mineral surfaces (Fig 3c). Even though there is a clear relationship between mineral stability and transfection, several mineral properties that have been reported to enhance transfection should be considered. For example, substrate topography effect on non-viral transfection has been studied through systematically controlled topographical library, indicating dense micropit patterns improve non-viral transfection of primary human cell type when compared with smooth substrates ${ }^{25}$. In addition, by measuring the level of $\mathrm{Ca}^{2+}$ change in cell culture medium during transfection, we confirmed that enhanced transfection was correlated to the mineral stability change (Supplementary Fig. S5). However, the level of a

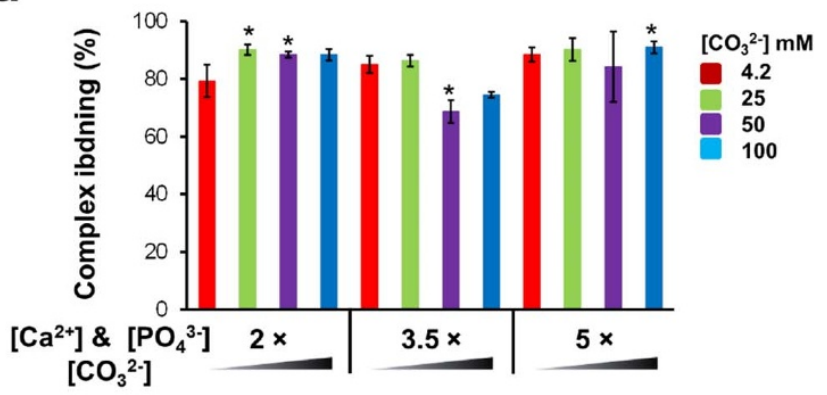

C

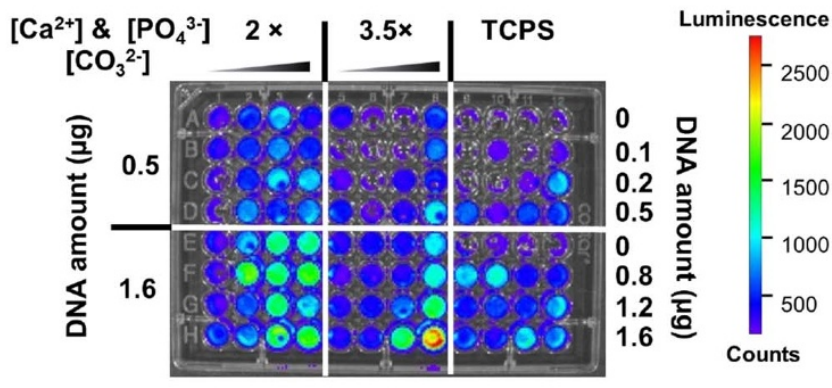

b

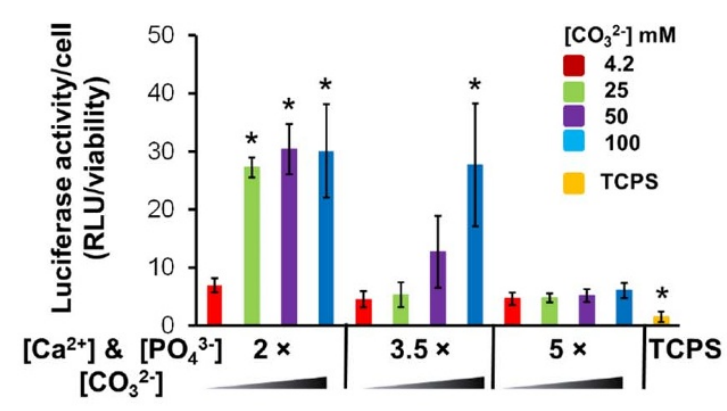

d

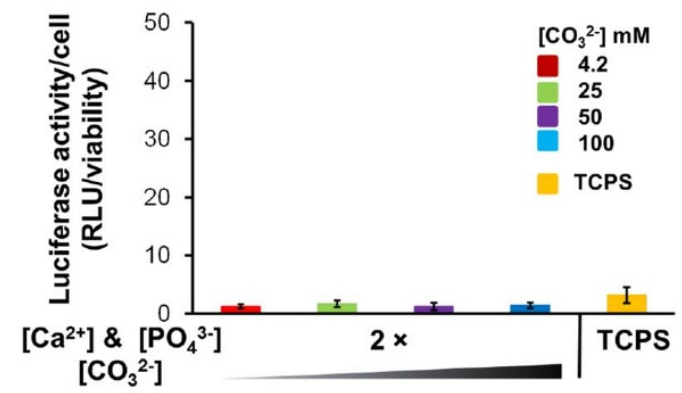

$\mathbf{e}$

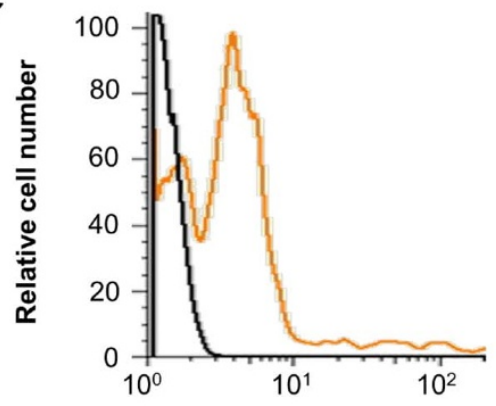

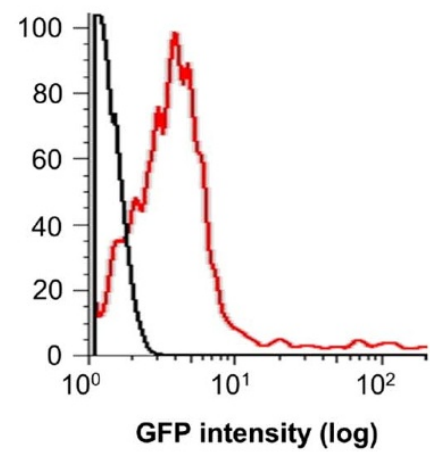

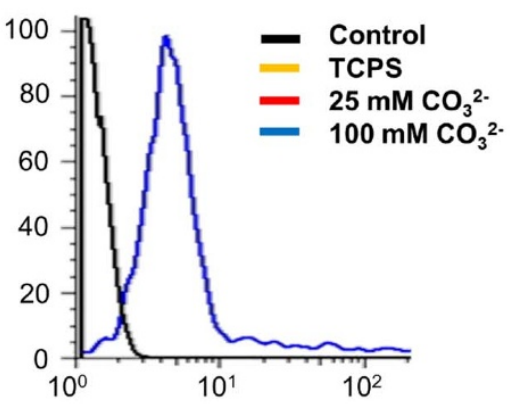

Figure 3 Screening of hMSC transfection on mineral coatings. (a) pDNA complexes binding on mineral coatings. The binding of the complexes was measured after $5 \mathrm{~h}$ incubation on the mineral coatings. ${ }^{*}$ indicates significant difference when compared with $\mathrm{Ca} / \mathrm{P}$ ratio of CaP mineral coating grown in $2 \times \mathrm{mSBF}$ with $4.2 \mathrm{mM}$ of $\mathrm{CO}_{3}{ }^{2-}$ (red bar at far left) by Student's $t$-test with $\mathrm{p}<0.05$. Data represents mean $\pm \mathrm{SD}(n=4)$. (b) Luciferase activity of hMSCs after 2 days culture on mineral coatings incubated with $3.2 \mu \mathrm{g}$ of pDNA complexes. To compare with a standard transfection method, soluble pDNA complexes were also added to hMSCs on tissue culture polystyrene (TCPS). ${ }^{*}$ indicates significant difference compared to $2 \times$ mSBF with $4.2 \mathrm{mM}$ of $\mathrm{CO}_{3}{ }^{2-}$ (red bar at far left) by Student's $t$-test with $\mathrm{p}<0.05$. Data represents mean $\pm \mathrm{SD}(n=4)$. (c) Luminescence intensity from cell culture medium after 2 days of hMSC transfection on mineral coatings and TCPS with different amounts of pDNA complexes. (d) Solution based delivery effect on transfection of hMSC on mineral coatings. pDNA complexes were added into cell culture medium after $1 \mathrm{~h}$ of cell seeding on mineral coatings formed in 2 $\times \mathrm{mSBF}$ with various $\left[\mathrm{CO}_{3}{ }^{2-}\right]$. For a control, hMSCs were grown on TCPS. Data represents mean $\pm \mathrm{SD}(n=6)$. (e) Flow cytometric analysis of the distribution of GFP positive cells after transfection on mineral coatings formed in $2 \times \mathrm{mSBF}$ with various $\left[\mathrm{CO}_{3}{ }^{2-}\right]$ and TCPS using pEGFP complexes. 
$\mathrm{PO}_{4}{ }^{3-}$ change was not proportional to mineral stability. This result may be due to differences in $\mathrm{Ca} / \mathrm{P}$ ratio of mineral coatings and the dynamics of mineral dissolution and re-precipitation. It should be noted that $\mathrm{pDNA}$ complex pre-loaded into mineral coatings provides enhanced transfection when compared with naked pDNA, as it is known that $\mathrm{pDNA}$ condensation provides size reduction for efficient uptake of pDNA and protection from nuclease attack (Supplementary Fig. S6). Notably, the effects of mineral coatings on non-viral transfection were only observed for substrate-mediated pDNA delivery, as luciferase activity of hMSCs on mineral coatings was not significantly improved when pDNA complexes were added in solution rather than pre-loaded into the coating (Fig. $3 \mathrm{~d}$ and Supplementary Fig. S7). This result emphasizes that the mechanism for enhanced non-viral transfection on inorganic coatings is related to the microenvironment between the cells and the mineral surfaces loaded with pDNA complexes.

Transfection screening for multiple plasmids and cell types. To validate the feasibility of changes in mineral properties for enhancing various target gene expression, we transfected hMSCs on mineral coatings loaded with pEGFP complexes. Result demonstrates two separate peaks of GFP positive cells on tissue culture polystyrene (TCPS). In contrast, there was a single, higher intensity peak of GFP positive cells with increasing $\mathrm{CO}_{3}{ }^{2-}$ content in the mineral coating (Fig. 3e), indicating that enhanced GFP expression is dependent on the stability of mineral coatings - similar to the results previously described for luciferase activity (Fig. 3b). The increased mean fluorescence intensity of GFP-positive cells also confirmed mineral stability effect on enhanced transfection on mineral coatings (Supplementary Fig. S8). Enhanced transfection induced by mineral stability change was further optimized by varying the amounts of pDNA complexes included within mineral coatings (Fig. 4 and Supplementary Fig. S9). In particular, combinatorial changes in mineral properties and pDNA complex amount identified conditions in which the luciferase activity per cell was increased by 68-fold for hMSCs, 56-fold for C3H10T1/2 cells, and 11-fold for primary human umbilical vein endothelial cells (HUVECs). These fold increases are each relative to standard manufacturer protocols using pDNA-Lipofectamine complexes in solution. In addition to the high levels of transfection relative to standard commercial techniques, combinatorial variation in mineral properties and pDNA complex amounts also resulted in controllable levels of transfection for each cell type characterized, and collectively the data show conditions in which the luciferase activity can be varied over a broad range of values. These results suggest that it will be practical to predictably vary the level of transfection in future studies by simply changing mineral coating properties.

Participation of calcium ion as a co-factor during transfection. To gain further insights into the role of $\mathrm{Ca}^{2+}$ in non-viral transfection on mineral coatings, we compared fluorescein-labeled pDNA complex uptake on surfaces with varying mineral coating stability, as cellular uptake is an important step for effective DNA transfer and has been previously shown to be influenced by $\mathrm{Ca}^{2+18}$. Within the fluorescein positive cell population, the data show two distributions of fluorescein positive cells (one peak at low intensity and a broad peak at higher intensity) on TCPS, and a single high intensity peak on mineral coatings. The position of the higher intensity fluorescein peak was shifted to increasing intensity regions with increasing $\mathrm{CO}_{3}{ }^{2-}$ content in the mineral coating (Fig. 5a). These results suggest that increased dissolution of mineral coatings (Fig. 2d) results in an increase in local $\left[\mathrm{Ca}^{2+}\right]$, which leads to enhanced uptake of pDNA complexes. This proposed mechanism is consistent with previous studies on the role of increased soluble $\left[\mathrm{Ca}^{2+}\right]$ in pDNA uptake ${ }^{18-20}$. Analysis of intracellular $\mathrm{Ca}^{2+}$ levels provided further insights into the role of $\mathrm{Ca}^{2+}$ during non-viral transfection on mineral coatings. Enhanced intracellular $\left[\mathrm{Ca}^{2+}\right]$ was observed
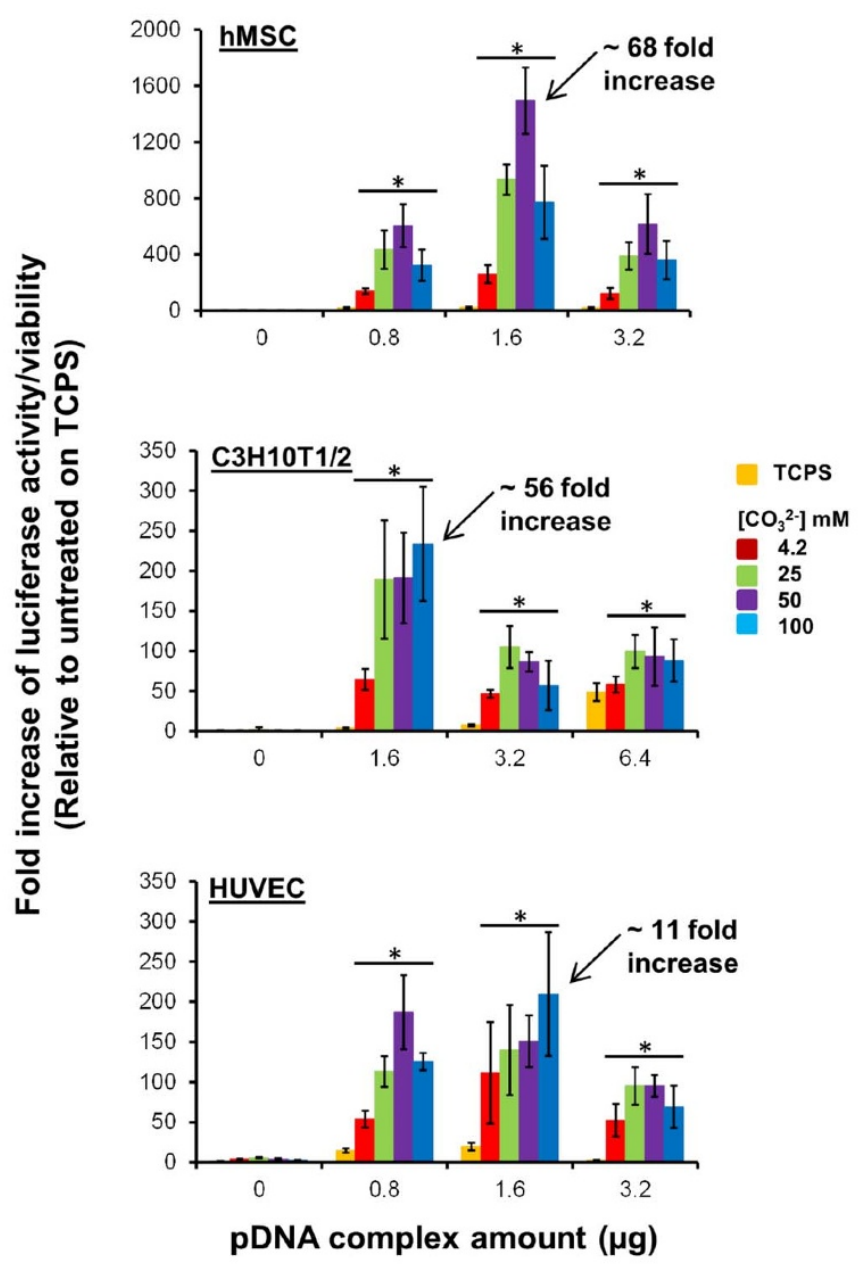

Figure $4 \mid$ Optimization of transfection of multiple cell types. Specified amounts of pDNA complexes were adsorbed on mineral coatings formed in $2 \times \mathrm{mSBF}$ with various $\left[\mathrm{CO}_{3}{ }^{2-}\right]$. Luciferase activity was measured after 2 days of transfection of hMSCs $(n=6), \mathrm{C} 3 \mathrm{H} 10 \mathrm{~T} 1 / 2$ cells $(n=3)$, and HUVECs $(n=6) . *$ indicates significant difference compared to TCPS condition by Student's $t$-test with $\mathrm{p}<0.05$. All data was normalized to TCPS with $0 \mu \mathrm{g}$ of pDNA complexes. Arrow indicates the condition that presents the highest luciferase activity calculated by normalizing the highest luciferase activity to the luciferase activity from TCPS at the same pDNA complexes amount. Luminescence was measured at different time point when compared with that measured in Figure 3.

when hMSCs were transfected on mineral surfaces that dissolved more rapidly. In contrast, intracellular $\left[\mathrm{Ca}^{2+}\right]$ levels in hMSCs transfected on TCPS using standard protocols were identical to levels in non-transfected hMSCs (Fig. 5b). Taken together, these results suggest that $\mathrm{Ca}^{2+}$ release from dissolving mineral coatings enhances uptake of pDNA complexes, and causes increases in intracellular $\left[\mathrm{Ca}^{2+}\right]$, which has previously been associated with enhanced endosomal escape of pDNA complexes ${ }^{18,21,22}$.

\section{Discussion}

This study shows enhanced and optimized non-viral transfection of multiple cell types by varying inorganic coating properties. Subtle changes in the properties of inorganic coatings resulted in widely variable transfection, and optimized coatings led to a 68-fold increase in transgene expression by stem cells when compared to standard techniques. A series of innovative previous studies have screened for the effects of libraries of organic molecules on non-viral transfection, including degradable poly (cations) ${ }^{16,26,27}$, peptoids ${ }^{28}$, and lipidoids ${ }^{29}$. 

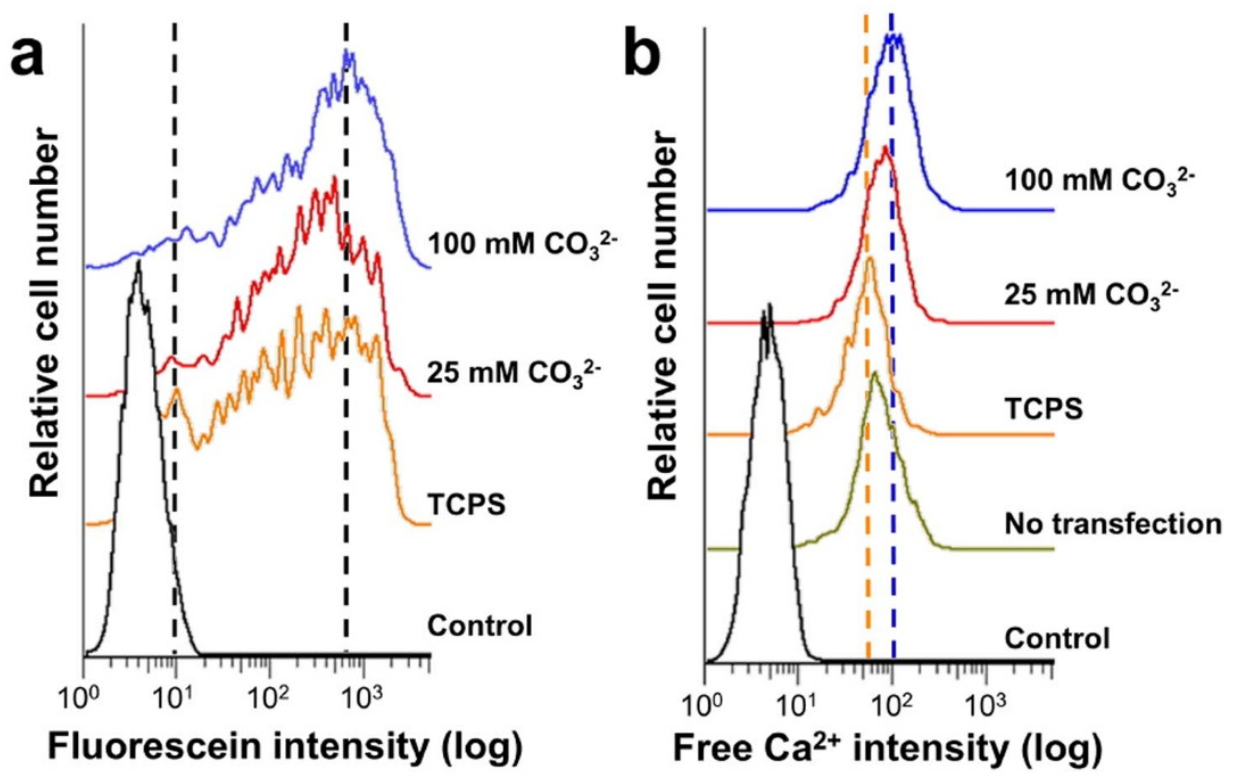

Figure 5 Possible mechanisms for enhanced transfection from mineral coatings. (a) Histogram of pDNA complexes uptaken by hMSC. Mineral coatings were formed in $2 \times \mathrm{mSBF}$ solutions. hMSCs were incubated on mineral coatings containing fluorescein-labeled pDNA complexes for 5 h. (Control: non-transfected cells grown on TCPS). (b) Intracellular calcium concentration after transfection of hMSCs. hMSCs were treated with a free $\mathrm{Ca}^{2+}$ indicator after transfection. (Control: non-transfected cells grown on TCPS without $\mathrm{Ca}^{2+}$ indicator; No transfection: non-transfected cells grown on TCPS with $\mathrm{Ca}^{2+}$ indicator).

These studies clearly demonstrated that efficient experimental formats can identify novel and superior non-viral vectors. The current study provides a first demonstration that inorganic materials can also be screened to optimize transfection. Analogous to the previous production of combinatorial libraries of organic non-viral vectors, the impact of this approach is that a library of structurally and compositionally distinct inorganic coatings can be generated in an efficient 96-well plate format. The resulting inorganic coatings serve as both a substrate for cell adhesion and a depot for localized release of non-viral vectors and calcium ions.

Efficient non-viral transfection requires pDNA from the extracellular space to overcome several cellular barriers, including DNA uptake across the cell membrane, endosomal escape, and nuclear entry. It is possible that calcium and phosphate ions released from dissolving mineral coatings can influence multiple stages of the transfection process. Previous studies have shown enhanced pDNA delivery using calcium as a main component of ionic complexes or as a cofactor with non-viral vectors ${ }^{19,30}$. In particular, the presence of soluble calcium ions during transfection has been shown to enhance transfection efficiency by facilitating DNA uptake ${ }^{18,19}$ and endosomal escape $^{20-22}$. In addition, calcium phosphate-pDNA nanoparticle complexes have been used for decades to enhance non-viral DNA delivery, as these complexes facilitate DNA uptake ${ }^{31,32}$. Here we found that calcium release from more rapidly dissolving mineral coatings led to increased intracellular calcium concentrations (Fig. 5b), as well as increased uptake of pDNA-Lipofectamine complexes (Fig. 5a). In turn, more rapidly dissolving mineral coatings supported enhanced transfection, and we can speculate that the increase in transfection is due in part to the role of locally released soluble calcium ions. It is also possible that calcium phosphatepDNA nanoparticles can form locally at the cell-substrate interface, resulting in enhanced local pDNA uptake. This possibility is supported by a previous study in which the addition of calcium ions in phosphate-free cell culture medium did not enhance transfection efficiency ${ }^{18}$.

Interestingly, enhanced transfection was only observed when pDNA complexes were released locally from the mineral coatings, and was abolished entirely when pDNA complexes were instead added to the cell culture medium in soluble form. Thus, the optimized transfection observed in this study is substrate-mediated, and related to the characteristics of the local environment near the surface of dissolving mineral coatings. Previous studies of locally immobilized pDNA complexes have emphasized that substrate-mediated pDNA delivery can lead to enhanced non-viral transfection ${ }^{33,34}$. The distinction here is that transfection is optimized by controlling the properties of inorganic coatings. The approach here is applicable to multiple pDNA complexes (Fig. 3) and multiple cell types (Fig. 4), which suggests a particularly broad applicability. It is also noteworthy that the library of inorganic coatings explored in this study can likely be readily expanded by controlled incorporation of other mineral ions known, including $\mathrm{F}^{-}$substitution for $\mathrm{OH}^{-}$ions (decreased coating dissolution) and $\mathrm{Mg}^{2+}$ substitution for $\mathrm{Ca}^{2+}$ ions (increased coating dissolution) ${ }^{35-38}$.

Inorganic coatings similar to those explored here have recently been formed on a variety of medical devices, including sutures ${ }^{39}$, screws $^{40}$, microspheres ${ }^{41}$, and tissue engineering scaffolds ${ }^{42}$. Thus, the approach described here may be particularly translatable to medical device applications where efficient non-viral transfection is desirable. To explore this applicability, we coated 3-dimensional PLG tissue engineering scaffolds in a 96-well format and explored non-viral transfection of hMSCs within scaffolds (Supplementary Fig. S10). Our results demonstrate increased hMSC transfection on scaffolds that were coated under the same conditions that promoted the highest hMSC transfection on 2-dimensional films (Supplementary Fig. S11). In addition, up scaling the coating process toward large scaffolds for a pig mandible transplant suggests that optimal coatings discovered in 2-dimensional formats can be translated to 3dimensional medical devices (Supplementary Fig. S12). These results provide promising evidence that enhanced non-viral transfection can be achieved in the context of a medical device application. However, a series of issues will need to be addressed to enable in vivo translation of these types of coated scaffolds, including the influence of dynamic in vivo environments on mineral coating stability and local cell behavior. 


\section{Methods}

Fabrication and characterization of mineral coatings. Poly (lactide-co-glycolide) (10 mg, PLG, lactide:glycolide $=85: 15$, average $\left.\mathrm{M}_{\mathrm{W}}=50,000-70,000\right)$ was dissolved in acetone and transferred in each well. Dried PLG was hydrolyzed in $\mathrm{NaOH}$ solution $(0.5 \mathrm{M})$ for $30 \mathrm{~min}$, then rinsed with DI water. Hydrolyzed PLG in each well was incubated in various mineral solutions for mineral formation (see SI Methods). The morphology of mineral coatings on each well was examined by SEM (Carl Zeiss SMT, model LEO-1530). The samples were coated with gold using a sputter coater (Denton Vacuum, model DESK II). The crystallinity index of mineral coatings was analyzed from spectra measured by FTIR spectrometer (Bruker, model EQUINOX 55) (see SI Supporting Information). To characterize mineral stability change, mineral coatings were incubated in DMEM (Mediatech, Manassas, VA) serum-free solution at $37^{\circ} \mathrm{C}$ with $\mathrm{pH}$ 7.4. To characterize the change of $\mathrm{Ca}^{2+}$ amount in DMEM solutions, DMEM solutions incubated with mineral coatings were assayed at specified times and replaced by fresh DMEM serum-free solution $(200 \mu \mathrm{L})$. To assay for soluble $\mathrm{Ca}^{2+}$ concentrations, working solution (0.4 mM Arsenazo III (MP Biomedicals, Solon, $\mathrm{OH}$ ) in $0.02 \mathrm{M}$ Tris-base at $\mathrm{pH}$ 7.4) was mixed with aliquot of each solution. Absorbance at $650 \mathrm{~nm}$ was converted to $\mathrm{Ca}^{2+}$ concentration using standard curves relating absorbance intensity to $\mathrm{Ca}^{2+}$ concentration

\section{Adsorption of pDNA-Lipofectamine 2000 complexes on mineral coatings.} pMetLuc and pEGFP-N1 reporter vectors were purchased from Clontech (Mount View, CA) and was amplified in competent TOF10F' E. coli (Invitrogen, Carlsbad, CA) and purified using a Mega plasmid purification kit (Qiagen, Valencia, CA) according to the manufacturer's protocol. Lipofectamine 2000 (Invitrogen, Carlsbad, $\mathrm{CA}$ ) was mixed with pMetLuc (N:P = 2.5) or pEGFP-N1 $(\mathrm{N}: \mathrm{P}=3)$ to form complexes in DMEM serum-free solution for $20 \mathrm{~min}$. Mineral coatings were incubated in DMEM serum-free solution $(60 \mu \mathrm{L})$ containing various amount of pDNA complexes for $5 \mathrm{~h}$ to allow the complexes to be adsorbed on mineral coatings.

Cell transfection on mineral coatings. Before seeding cells on mineral coatings containing complexes, Cells (hMSC, C3H10T1/2 and HUVEC) cells were grown on flask at $37^{\circ} \mathrm{C}$ and $5 \% \mathrm{CO}_{2}$ in different media (hMSC: MEM (Mediatech, Manassas, VA) with $10 \%$ fetal bovine serum (FBS) and $1 \%$ penicillin/streptomycin (P/S); C3H10T1/2: DMEM with 10\% cosmic calf serum (CCS) and 1\% P/S; HUVEC: M199 with $2 \% \mathrm{EGM}-2,20 \% \mathrm{FBS}$, and $1 \% \mathrm{P} / \mathrm{S}$ ) respectively. At the time of transfection, cells were collected by treatment with $0.05 \%$ trypsin-EDTA $(10 \mathrm{~mL})$ and seeded on the mineral coatings containing complexes at the density of $3.3 \times 10^{4} \mathrm{cells} / \mathrm{cm}^{2}$ and cultured in the media, containing HEPES $(25 \mathrm{mM})$. For transfection on TCPS, cells were grown one day before transfection and transfected by adding complexes into cell culture media. After $2 \mathrm{~d}$ of cell culture on mineral coatings and TCPS, each medium was taken for luciferase activity assay and refreshed with fresh medium $(100 \mu \mathrm{L})$ for cell viability assay.

Luminescence and cell viability assays. For measuring luciferase activity, sample solutions $(50 \mu \mathrm{L})$ after $2 \mathrm{~d}$ of transfection was mixed with working solution $(5 \mu \mathrm{L})$ prepared using the Cell-Glow Luciferase assay kit (Clontech, Mountain view, CA). Luminescence from secreted luciferase was measured using microplate luminometer (Veritas, Sunnyvale, CA) after $10 \mathrm{~min}$ of adding working solution. To calculate luciferase activity per viable cell, luminescence was divided by cell viability measured using CellTiter-Blue cell viability assay kit (Promega, Madison, WI). Also, luminescence per well was imaged using in vivo imaging system (IVIS) (Caliper Life Sciences, Mountain view, CA) after 5 min of adding working solution.

Flow cytometry. For GFP expression measurement, hMSC cells transfected on mineral coatings containing pEGFP complexes were removed with 0.05\% trypsinEDTA after $2 \mathrm{~d}$ of transfection. Cells were washed and re-suspended in PBS containing $1 \%$ FBS. $1 \times 10^{4}$ cells were analyzed for each sample with FACSCalibur (BD). Untrasfected cells were used as control to set live single-cell population and auto-fluorescence. Gated live single-cell population was used to analyze GFP positive cells from mineral coatings and tissue culture polystyrene (TCPS). Data analysis was performed using FlowJo software (Treestar, Inc.). For uptake of pDNA complexes, pMetLuc was labeled with fluorescein labeling kit (Mirus Bio). After $5 \mathrm{~h}$ of transfection on mineral coatings containing fluorescein-labeled pDNA complexes, cells were removed with $0.05 \%$ trypsin-EDTA and wash in PBS containing $1 \%$ FBS and then re-suspended in $0.4 \%$ trypan blue to quench the fluorescence from complexes adsorbed on cell surface. Cells were washed twice and re-suspended with PBS containing 1\% FBS. To analyze intracellular $\mathrm{Ca}^{2+}$ concentration, hMSC cells transfected for $5 \mathrm{~h}$ were removed and re-suspended in MEM serum-free solution containing Calcium Green ${ }^{\mathrm{TM}}-5 \mathrm{~N}(4 \mu \mathrm{M})$ (Molecular Probes). After 20 min of incubation at RT, cells were washed twice with PBS containing $1 \%$ FBS and then immediately analyzed. Untransfected cells without treatment with calcium indicator were used as control.

1. Kagan, C. R., Mitzi, D. B. \& Dimitrakopoulos, C. D. Organic-inorganic hybrid materials as semiconducting channels in thin-film field-effect transistors. Science 286, 945-947 (1999).

2. Yang, Y., Kim, K. H. \& Ong, J. L. A review on calcium phosphate coatings produced using a sputtering process-an alternative to plasma spraying. Biomaterials 26, 327-337 (2005).
3. Bunker, B. C. et al. Ceramic thin-film formation on functionalized interfaces through biomimetic processing. Science 264, 48-55 (1994).

4. Yan, W. Q. et al. Apatite layer-coated titanium for use as bone bonding implants. Biomaterials 18, 1185-1190 (1997).

5. Du, C. et al. Biomimetic calcium phosphate coatings on Polyactive ${ }^{\circledR} 1000 / 70 / 30$. J. Biomed. Mater. Res. 59, 535-546 (2002).

6. Majid, K., Tseng, M. D., Baker, K. C., Reyes-Trocchia, A. \& Herkowitz, H. N. Biomimetic calcium phosphate coatings as bone morphogenetic protein delivery systems in spinal fusion. Spine J. 11, 560-567 (2011).

7. Shen, H., Tan, J. \& Saltzman, W. M. Surface-mediated gene transfer from nanocomposites of controlled texture. Nat. Mater. 3, 569-574 (2004).

8. Dorozhkina, E. I. \& Dorozhkin, S. V. Structure and properties of the precipitates formed from condensed solutions of the revised simulated body fluid. J. Biomed. Mater. Res. A 67A, 578-581 (2003).

9. Shin, K., Jayasuriya, A. C. \& Kohn, D. H. Effect of ionic activity products on the structure and composition of mineral self assembled on three-dimensional poly(lactide-co-glycolide) scaffolds. J. Biomed. Mater. Res. A 83A, 1076-1086 (2007).

10. Müller, L. \& Müller, F. A. Preparation of SBF with different content and its influence on the composition of biomimetic apatites. Acta Biomater. 2, 181-189 (2006).

11. Pan, H. \& Darvell, B. W. Effect of carbonate on hydroxyapatite solubility. Cryst. Growth Des. 10, 845-850 (2010).

12. Costa, D. O. et al. Control of surface topography in biomimetic calcium phosphate coatings. Langmuir 28, 3871-3880 (2012).

13. Ban, S. \& Maruno, S. Morphology and microstructure of electrochemically deposited calcium phosphates in a modified simulated body fluid. Biomaterials 19, 1245-1253 (1998).

14. Chou, Y. F., Huang, W., Dunn, J. C. Y., Miller, T. A. \& Wu, B. M. The effect of biomimetic apatite structure on osteoblast viability, proliferation, and gene expression. Biomaterials 26, 285-295 (2005).

15. Sun, B., Tran, K. K. \& Shen, H. Enabling customization of non-viral gene delivery systems for individual cell types by surface-induced mineralization. Biomaterials 30, 6386-6393 (2009)

16. Lynn, D. M., Anderson, D. G., Putnam, D. \& Langer, R. Accelerated discovery of synthetic transfection vectors: parallel synthesis and screening of a degradable polymer library. J. Am. Chem. Soc. 32, 8155-8156 (2001).

17. Hilbrig, F. \& Freitag, R. Isolation and purification of recombinant proteins, antibodies and plasmid DNA with hydroxyapatite chromatography. Biotechnol. J. 7, 90-102 (2012).

18. Sandhu, A. P. et al. Calcium enhances the transfection potency of stabilized plasmid-lipid particles. Anal. Biochem. 341, 156-164 (2005).

19. Lam, A. M. I. \& Cullis, P. R. Calcium enhances the transfection potency of plasmid DNA-cationic liposome complexes. BBA - Biomembranes 1463, 279-290 (2000).

20. Gerasimenko, J. V., Tepikin, A. V., Petersen, O. H. \& Gerasimenko, O. V. Calcium uptake via endocytosis with rapid release from acidifying endosomes. Curr. Biol. 8 , 1335-1338 (1998).

21. Haberland, A. et al. Calcium ions as efficient cofactor of polycation-mediated gene transfer. BBA - Gene Struct. Expr. 1445, 21-30 (1999).

22. Fujita, T. et al. Calcium enhanced delivery of tetraarginine-PEG-lipid-coated DNA/protamine complexes. Int. J. Pharm. 368, 186-192 (2009).

23. Murphy, W. L. \& Mooney, D. J. Bioinspired growth of crystalline carbonate apatite on biodegradable polymer substrata. J. Am. Chem. Soc. 124, 1910-1917 (2002).

24. Chou, Y. F., Chiou, W. A., Xu, Y., Dunn, J. C. Y. \& Wu, B. M. The effect of pH on the structural evolution of accelerated biomimetic apatite. Biomaterials 25, 5323-5331 (2004).

25. Adler, A. F. et al. High-throughput screening of microscale pitted substrate topographies for enhanced nonviral transfection efficiency in primary human fibroblasts. Biomaterials 32, 3611-3619 (2011).

26. Thomas, M., Lu, J., Zhang, C., Chen, J. \& Klibanov, A. Identification of novel superior polycationic vectors for gene delivery by high-throughput synthesis and screening of a combinatorial library. Pharm. Res. 24, 1564-1571 (2007).

27. Yingyongnarongkul, B. E., Howarth, M., Elliott, T. \& Bradley, M. Solid-phase synthesis of 89 polyamine-based cationic lipids for DNA delivery to mammalian cells. Chem. - Eur. J. 10, 463-473 (2004).

28. Murphy, J. E. et al. A combinatorial approach to the discovery of efficient cationic peptoid reagents for gene delivery. Proc. Natl. Acad. Sci. USA 95, 1517-1522 (1998).

29. Akinc, A. et al. A combinatorial library of lipid-like materials for delivery of RNAi therapeutics. Nat. Biotech. 26, 561-569 (2008).

30. Jordan, M., Schallhorn, A. \& Wurm, F. M. Transfecting mammalian cells: optimization of critical parameters affecting calcium-phosphate precipitate formation. Nucleic Acids Res. 24, 596-601 (1996).

31. Cao, X. et al. Encapsulation of plasmid DNA in calcium phosphate nanoparticles: stem cell uptake and gene transfer efficiency. Int. J. Nanomed. 6, 3335-3349 (2011).

32. Sokolova, V., Kovtun, A., Heumann, R. \& Epple, M. Tracking the pathway of calcium phosphate/DNA nanoparticles during cell transfection by incorporation of red-fluorescing tetramethylrhodamine isothiocyanate-bovine serum albumin into these nanoparticles. J. Biol. Inorg. Chem. 12, 174-179 (2007).

33. Segura, T. \& Shea, L. D. Surface-tethered DNA complexes for enhanced gene delivery. Bioconjugate Chem. 13, 621-629 (2002). 
34. Jang, J. H., Bengali, Z., Houchin, T. L. \& Shea, L. D. Surface adsorption of DNA to tissue engineering scaffolds for efficient gene delivery. J. Biomed. Mater. Res. A 77A, 50-58 (2006)

35. Driessens, F. C. M. Relation between apatite solubility and anti-cariogenic effect of fluoride. Nature 243, 420-421 (1973).

36. Moreno, E. C., Kresak, M. \& Zahradnik, R. T. Fluoridated hydroxyapatite solubility and caries formation. Nature 247, 64-65 (1974).

37. Chowdhury, E. H. et al. High-efficiency gene delivery for expression in mammalian cells by nanoprecipitates of $\mathrm{Ca}-\mathrm{Mg}$ phosphate. Gene 341, 77-82 (2004)

38. Barrere, F., van Blitterswijk, C. A., de Groot, K. \& Layrolle, P. Nucleation of biomimetic Ca-P coatings on Ti6Al4V from a SBF $\times 5$ solution: influence of magnesium. Biomaterials 23, 2211-2220 (2002)

39. Lee, J. S., Lu, Y., Baer, G. S., Markel, M. D. \& Murphy, W. L. Controllable protein delivery from coated surgical sutures. J. Mater. Chem. 20, 8894-8903 (2010)

40. Lu, Y. et al. Influence of hydroxyapatite-coated and growth factor-releasing interference screws on tendon-bone healing in an ovine model. Arthroscopy 25, 1427-1434 (2009).

41. Jongpaiboonkit, L., Franklin-Ford, T. \& Murphy, W. L. Mineral-coated polymer microspheres for controlled protein binding and release. Adv. Mater. 21, 1960-1963 (2009)

42. Nandakumar, A., Yang, L., Habibovic, P. \& van Blitterswijk, C. Calcium phosphate coated electrospun fiber matrices as scaffolds for bone tissue engineering. Langmuir 26, 7380-7387 (2009).

\section{Acknowledgments}

The authors are grateful for valuable comments with M. Schwartz and J. S. Lee. This research was supported by the AO Research Foundation (Exploratory Research Grant) and the National Institutes of Health (R01AR059916).

\section{Author contributions}

S.C. and W.M. designed the experiments wrote the manuscript. S.C. performed and analyzed all 2-dimentional experiments. X.H. and L.J. carried out and analyzed 3-dimentional scaffolds experiments. W.M. and S.H. contributed to the interpretation and reviewed the manuscript.

\section{Additional information}

Supplementary information accompanies this paper at http://www.nature.com/ scientificreports

Competing financial interests: Co-authors Murphy and Hollister are co-founders and shareholders in Tissue Regeneration Systems, Inc., the company that is developing mandibular reconstruction devices presented in Supplementary Figure S12. Co-author Jongpaiboonkit is an employee of Tissue Regeneration Systems, Inc.

License: This work is licensed under a Creative Commons

Attribution-NonCommercial-NoDerivs 3.0 Unported License. To view a copy of this license, visit http://creativecommons.org/licenses/by-nc-nd/3.0/

How to cite this article: Choi, S., Yu, X., Jongpaiboonkit, L., Hollister, S.J. \& Murphy, W.L Inorganic coatings for optimized non-viral transfection of stem cells. Sci. Rep. 3, 1567; DOI:10.1038/srep01567 (2013) 